\title{
Increased expression of the receptor for advanced glycation end products in neurons and astrocytes in a triple transgenic mouse model of Alzheimer's disease
}

\begin{abstract}
Bo-Ryoung Choi ${ }^{1,4}$, Woo-Hyun Cho ${ }^{1,4}$, Jiyoung Kim² ${ }^{2}$ Hyong Joo Lee ${ }^{2}$, ChiHye Chung ${ }^{1}$, Won Kyung Jeon ${ }^{3}$ and Jung-Soo Han ${ }^{1}$

The receptor for advanced glycation end products (RAGE) has been reported to have a pivotal role in the pathogenesis of Alzheimer's disease (AD). This study investigated RAGE levels in the hippocampus and cortex of a triple transgenic mouse model of AD (3xTg-AD) using western blotting and immunohistochemical double-labeling to assess cellular localization. Analysis of western blots showed that there were no differences in the hippocampal and cortical RAGE levels in 10-month-old adult 3xTg-AD mice, but significant increases in RAGE expression were found in the 22- to 24-month-old aged 3xTg-AD mice compared with those of age-matched controls. RAGE-positive immunoreactivity was observed primarily in neurons of aged 3XTg-AD mice with very little labeling in non-neuronal cells, with the notable exception of RAGE presence in astrocytes in the hippocampal area CA1. In addition, RAGE signals were co-localized with the intracellular amyloid precursor protein (APP)/ amyloid beta (A $\beta$ ) but not with the extracellular APP/A $\beta$. In aged 3xTg-AD mice, expression of human tau was observed in the hippocampal area CA1 and co-localized with RAGE signals. The increased presence of RAGE in the 3xTg-AD animal model showing critical aspects of $A D$ neuropathology indicates that RAGE may contribute to cellular dysfunction in the AD brain. Experimental \& Molecular Medicine (2014) 46, e75; doi:10.1038/emm.2013.147; published online 7 February 2014
\end{abstract}

Keywords: Alzheimer's disease; astrocyte; cortex; hippocampus; mice; receptor for advanced glycation end products (RAGE)

\section{INTRODUCTION}

Alzheimer's disease $(\mathrm{AD})$ is an age-related disorder characterized by cognitive impairments. ${ }^{1,2}$ Two major pathological characteristics of $\mathrm{AD}$ are the formation of senile plaques (SPs) and numerous neurofibrillary tangles (NFTs). Deposition of amyloid beta $(\mathrm{A} \beta)$, the main component of SPs, is found in extracellular SPs, although accumulation of intraneuronal $A \beta$ is also observed in patients with $\mathrm{AD}$ and in animal models for AD. ${ }^{2-5}$ NFTs are composed of hyperphosphorylated tau. ${ }^{6}$

The receptor for advanced glycation end products (RAGE), a multiligand receptor belonging to the immunoglobulin superfamily, functions as a cell surface-binding site for advanced glycation end products (AGEs), which are adducts resulting from non-enzymatic glycation and oxidation of lipids, proteins and nucleic acids. ${ }^{7}$ In addition, RAGE acts as a receptor for $\mathrm{A} \beta$, and its expression level is increased in $\mathrm{AD}$ brains, indicating that it has a role in the pathogenesis of neuronal dysfunction. ${ }^{8}$ Recent studies implicate a role for RAGE in mediating the effects of AGEs on tau phosphorylation. ${ }^{9,10}$

Previous reports have examined the distribution and expression levels of RAGE using the brain tissue from patients with $\mathrm{AD}$ and control patients without dementia. RAGE expression levels in $\mathrm{AD}$ brains were increased compared with control brains, and RAGE was shown to be present in neurons, glia and microglia in the hippocampus and cortex. ${ }^{11-13}$ Studies employing transgenic animals with targeted neuronal overexpression of a mutant amyloid precursor protein (APP) have reported that the increased expression of RAGE exacerbated the changes caused by mutant APP (that is, impairment of memory and of synaptic plasticity and

${ }^{1}$ Department of Biological Sciences, Konkuk University, Seoul, Republic of Korea; ${ }^{2}$ WCU Biomodulation Major, Department of Agricultural Biotechnology, Seoul National University, Seoul, Republic of Korea and ${ }^{3}$ Herbal Medicine Research Division, Korea Institute of Oriental Medicine, Daejeon, Republic of Korea

${ }^{4}$ These authors contributed equally to this work.

Correspondence: Professor J-S Han, Department of Biological Sciences, Konkuk University, 1 Hwayang-dong, Kwangjin-Gu, Seoul 143-701, Republic of Korea. E-mail: jshan06@konkuk.ac.kr

or Dr WK Jeon, Herbal Medicine Research Division, Korea Institute of Oriental Medicine, Daejeon, Republic of Korea.

E-mail: wkjeon@kiom.re.kr

Received 8 September 2013; revised 25 October 2013; accepted 30 October 2013 
neuropathologic markers). Conversely, experimental treatments that suppress RAGE expression in transgenic mice with an $A \beta$ rich environment prevent memory impairment and diminish neuropathologic changes. ${ }^{14,15}$ These results indicate that RAGE is a facilitator for $\mathrm{A} \beta$-induced neuropathologic changes.

In addition to plaque formation, NFTs are involved in the $\mathrm{AD}$ neurodegenerative process. ${ }^{16}$ Paired helical filament tau in NFTs is co-localized with AGEs, another ligand of RAGE. ${ }^{10} \mathrm{~A}$ recent study demonstrated that exogenous infusion of AGEs induces tau hyperphosphorylation and memory impairment through RAGE-mediated glycogen synthase kinase- 3 activity. ${ }^{9}$ Thus, expression of RAGE might be influenced by $A \beta$ and tau in the $\mathrm{AD}$ neurodegenerative process. Therefore, the present study was conducted to examine the expression levels and cellular localization of RAGE and the degree of co-localization between RAGE and $\mathrm{A} \beta$ or tau in the hippocampus and cortex of triple transgenic $\mathrm{AD}$ (3xTg-AD) mice. This is a mouse model of AD that overexpresses human tau and APP in a mutant presenilin1 knock-in background and shows AD-like SPs and NFTs, thus exhibiting the full spectrum of $\mathrm{AD}$ neurology. 5,17

\section{MATERIALS AND METHODS}

\section{Subjects}

Female adult (10 months old) and aged (22-24 months old) 3xTg-AD mice (adult: $n=6$; aged: $n=10$ ) and their non-Tg control littermates (adult: $n=6$; aged: $n=14$ ) were used. Transgenic and non-Tg breeding pairs were kindly provided by Dr Frank LaFerla from the University of California Irvine (Irvine, CA, USA). The generation of 3xTg-AD mice has been reported previously., ${ }^{5,17,18}$ Genotypes were determined by polymerase chain reaction analysis of DNA obtained from tail biopsies. Mice were housed in groups of four per cage and were placed in a temperature- and humidity-controlled room with a 12-h light/dark cycle (lights on from 0700 to 1900 hours). Food and water were available ad libitum. Experiments were conducted in compliance with the Konkuk University's Council Directive for the use and care of laboratory animals.

\section{Western blot analysis}

Young and aged $3 \times \mathrm{Tg}-\mathrm{AD}$ and non-Tg control mice $(n=6$ per each group) were anesthetized with an overdose of ketamine $\left(100 \mathrm{mg} \mathrm{kg}^{-1}\right)$ and xylazine $\left(5 \mathrm{mg} \mathrm{kg}^{-1}\right)$. The mice were then intracardially perfused with $0.1 \mathrm{M}$ phosphate-buffered saline $(\mathrm{pH}$ 7.4) to remove RAGE in the blood. Following perfusion, the brains were removed, and the hippocampus and cortex were rapidly dissected and frozen at $-80^{\circ} \mathrm{C}$ until further processing. Proteins for RAGE analysis were extracted in the following manner. Individual tissue samples were weighed and then homogenized in ice-cold buffer $\left(0.2 \mathrm{mg} \mathrm{ml}^{-1}\right)$ containing $20 \mathrm{~mm}$ Tris, $\mathrm{pH} 7.5,5 \%$ glycerol, $1.5 \mathrm{~mm}$ ethylenediaminetetraacetic acid, $40 \mathrm{~mm} \mathrm{KCl}, 0.5 \mathrm{~mm}$ dithiothreitol and protease inhibitors (No. 539131, Calbiochem, Darmstadt, Germany). Homogenates were centrifuged at $18000 \mathrm{~g}$ for $1 \mathrm{~h}$ at $4{ }^{\circ} \mathrm{C}$. The supernatant was removed from each sample, and an aliquot was taken for determination of total protein concentration using the Bradford reagent. The proteins were then separated by sodium dodecyl sulfate-polyacrylamide gel electrophoresis and transferred to a polyvinylidene difluoride membrane. The membrane was incubated with a primary antibody $(\mathrm{Ab})$ against RAGE (1:2000, Thermo
Scientific, Waltham, MA, USA, $45 \mathrm{kDa}$ ), which was used to detect the RAGE signal of the brain in previous studies. ${ }^{19,20}$ Following primary incubation, blots were incubated with the horseradish peroxidase-conjugated secondary Ab (1:2000, Cell Signaling, Danvers, MA, USA). Blots were visualized using an enhanced chemiluminescence system and developed using Hyperfilm (Amersham, Piscataway, NJ, USA). The relative expression level of RAGE was determined by densitometry and normalization to $\beta$-actin (1:5000, Sigma, St Louis, MO, USA), an invariant cytoskeletal protein.

\section{Immunohistochemistry}

Aged $3 \times \operatorname{Tg}-\mathrm{AD} \quad(n=4)$ and non-Tg control mice $(n=8)$ were intracardially perfused with ice-cold $0.01 \mathrm{M}$ phosphate-buffered saline and subsequently $4 \%$ paraformaldehyde under deep anesthesia. The brain was then removed and post-fixed in $4 \%$ paraformaldehyde for 2 days, cryoprotected in phosphate-buffered saline containing 30\% sucrose for $48 \mathrm{~h}$, frozen on powdered dry ice and sectioned using a microtome.

Coronal sections containing the hippocampus or cortex were used for evaluating RAGE co-localization with neuron, glia and AD-related markers (bregma anterior-posterior, -3.12 to $-4.56 \mathrm{~mm}$ for the hippocampus; +1.7 to $+0.5 \mathrm{~mm}$ for the cortex). Sections were blocked with $10 \%$ fetal horse serum (GIBCO, Carlsbad, CA, USA) with $3 \%$ triton X-100 in phosphate-buffered saline for $1 \mathrm{~h}$. Sections were then incubated in the appropriate primary antibody solution. For RAGE co-localization, mouse anti-NeuN antibody (Millipore, Darmstadt, Germany; 1:1000), rabbit anti-Iba-1 antibody (Wako, Osaka, Japan; 1:1000) and mouse anti-GFAP antibody (BD Bioscience, San Jose, CA, USA; 1:1000) were used with goat antiRAGE antibody (Serotec, Raleigh, NC, USA; 1:1000) or rabbit antiRAGE antibody (ABR, Golden, CO, USA; 1:1000). For AD-related marker co-localization, mouse anti-beta amyloid (4G8) antibody (Covance, Princeton, NJ, USA; 1:1000), mouse anti-tau (HT7) antibody (Thermo Scientific, 1:200), goat anti-RAGE antibody (Serotec, 1:1000) and rabbit anti-RAGE antibody (ABR, 1:1000) were used. Sections were incubated in primary antibody solution for $20 \mathrm{~h}$ at room temperature. After subsequent washing, the sections were incubated in Alexa dye-conjugated secondary antibody solution including Alexa 488 conjugated donkey anti-mouse antibody, Alexa 568-conjugated donkey anti-rabbit antibody and/or Alexa 633-conjugated donkey anti-goat antibody (Invitrogen, Carlsbad, CA, USA; 1:200). After staining, sections were mounted onto silane-coated glasses and coverslipped with ProLong Gold Antifade reagent (Invitrogen). Before observing the sections, stained sections were kept in the freezer at $-20^{\circ} \mathrm{C}$. Stained sections were observed under a confocal microscope (Olympus, Tokyo, Japan) and signal intensity was integrated to measure fluorescence signal density strength with the FluoView 1000 confocal microscope analysis program FV10-ASW 2.0 (Olympus). Five regions of interest (ROI) were selected within the cortex, each measuring $5000 \mu^{2}$. These ROIs contained all cortical layers in each hemisphere, and both hemispheres in one section were used for quantification. An ROI containing the fifth layer of the cortex was analyzed separately, and the other four ROIs contained all layers except the fifth layer. Five ROIs were also selected within the hippocampus, each measuring $3000 \mu \mathrm{m}^{2}$. These ROIs were drawn within the CA1, CA3 and dentate gyrus areas of the hippocampus.

\section{Statistical analysis}

Western blot data were analyzed by two-way analysis of variance (ANOVA) with group (non-Tg control vs 3xTg-AD) and age (adult vs 
aged) to examine the levels of RAGE. Immunoreactivity data from immunohistochemistry experiments were analyzed by two-way ANOVA with group (non-Tg control vs $3 \mathrm{xTg}-\mathrm{AD}$ ) and region (cortex and hippocampal subregions). Post hoc comparisons used Student's $t$-tests to assess differences between non-Tg control and 3xTg-AD groups. Any $P$-values that were less than 0.05 were considered significant. All data are expressed as the mean \pm s.e.m.

\section{RESULTS}

Increased RAGE levels in the hippocampus and cortex of aged 3xTg-AD mice

Many studies have reported that patients with $\mathrm{AD}$ have increased RAGE $(45 \mathrm{kDa})$ levels in the brains compared with aged-matched controls. ${ }^{12,13,21}$ Similarly, RAGE levels are higher in the brains of 24-month-old Tg2576 mice, an animal model for $\mathrm{AD}$ with overexpression of the Swedish mutant form of APP, which is linked to early-onset familial AD. ${ }^{15}$ However, mouse models of $\mathrm{AD}$ expressing mutant forms of APP or PS are limited in that they do not exhibit the full spectrum of $\mathrm{AD}$ pathologies and characteristics. Therefore, the present experiment examined alterations of RAGE in the $3 \mathrm{xTg}-\mathrm{AD}$ model, which was constructed to overcome this limitation.

Protein levels of RAGE in the hippocampus and cortex of adult (10 months old) and aged (22-24 months old) 3xTg-AD mice and age-matched non-Tg control mice were examined using western blot analysis. Figure 1 shows the RAGE immunoblots for the hippocampus and cortex. Two-way ANOVA of the hippocampal levels of RAGE showed that the main effects of group were not significant $(F(1,20)=3.268$, $P=0.09)$, but the main effects of age were significant $(\mathrm{F}(1,20)=6.78, \quad P<0.01)$, and there was a significant interaction effect of age and group $(\mathrm{F}(1,20)=9.48, P<0.01)$. The post hoc analyses showed that in the hippocampus, the aged 3xTg-AD mice expressed increased levels of RAGE compared with the other groups $(P<0.05)$. Statistical analysis of RAGE levels in the cortex exhibited a similar pattern of results. Specifically, two-way ANOVA of RAGE levels in the cortex showed that the main effects of group and the interaction effects of group and age were not significant $(\mathrm{F}(1,20)<2.948, P \geqslant 0.10)$, but the main effects of age were significant $(\mathrm{F}(1,20)=9.87, P<0.01)$. Post hoc analyses showed that the cortical RAGE levels of aged $3 \times \mathrm{Tg}-\mathrm{AD}$ mice were significantly higher than those of the other groups $(P<0.05)$ and differences between the other groups were not significant. Taken together, these results indicate that age is a key determinant in the effects of group on RAGE expression levels.

Distribution and cellular localization of RAGE-positive cells RAGE expression occurs in neurons, microglia and endothelial cells in the brain. ${ }^{22}$ Using double-labeled fluorescent immunohistochemistry with human brain tissue, including those of patients with $\mathrm{AD}$, RAGE expression was shown to be expressed in neurons, glial cells and microglial cells. ${ }^{12,13,21}$ To our knowledge, no study has examined the cellular localization of RAGE in transgenic AD mice such as the 3xTg-AD mouse. Therefore, the present study examined the distribution and cellular localization of RAGE in the hippocampus and cortex in aged 3xTg-AD mice.

Immunohistochemistry was used to determine the pattern of RAGE expression in aged 3xTg-AD mice (Figure 2a). Consistent with western blot results, increased RAGE immunoreactivity was found in aged $3 x T g-A D$ mice. Two-way
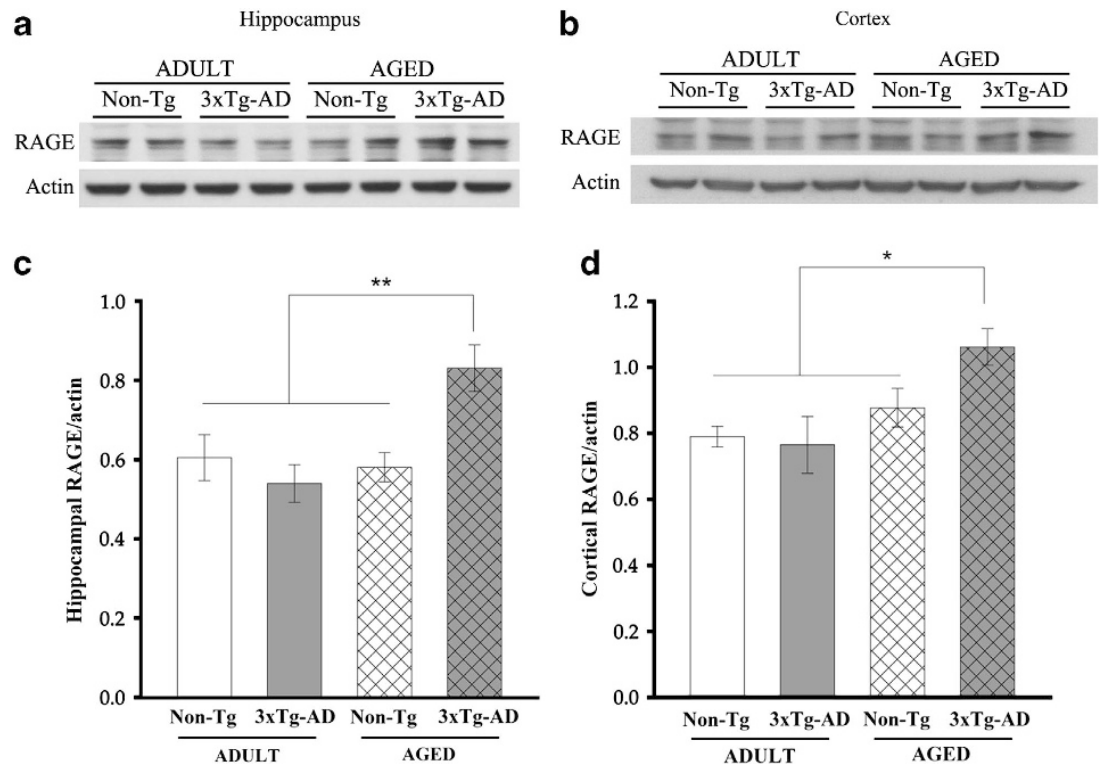

Figure 1 Levels of hippocampal and cortical RAGE in adult and aged 3xTg-AD and non-Tg control mice. Representative immunoblots of RAGE (45 kDa) expression in the hippocampus (a) and cortex (b) in adult and aged 3xTg-AD and non-Tg control mice. Levels of RAGE were significantly higher in aged 3xTg-AD mice than in aged non-Tg control, adult 3xTg-AD and adult non-Tg control mice (c, d). Data are expressed as the ratios of RAGE/actin (means \pm s.e.m.; $n=6$ per each group). ${ }^{*} P<0.05,{ }^{* *} P<0.01$. 

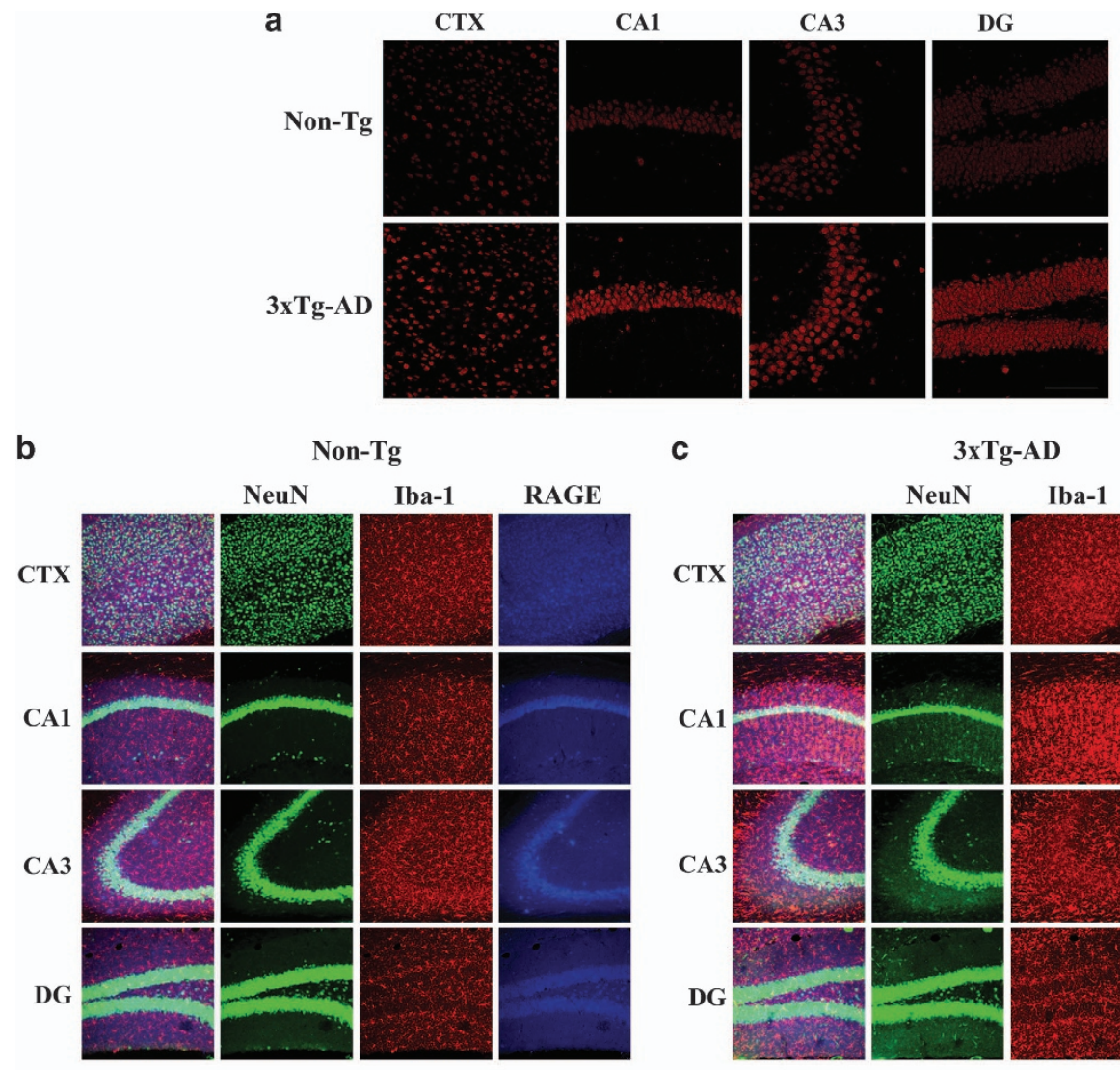

Non-Tg

c

3xTg-AD


Iba-1

RAGE
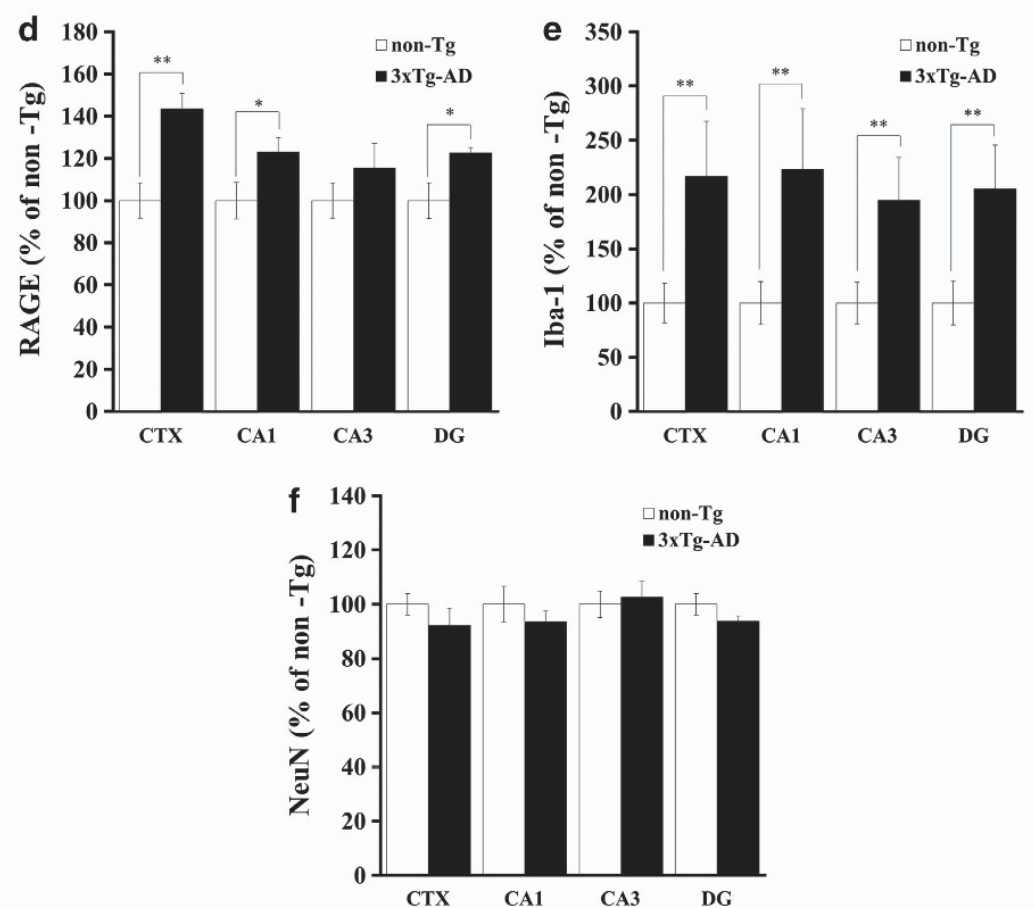

Figure 2 Distribution and cellular localization of RAGE in aged 3xTg-AD. Immunofluorescent RAGE images in the hippocampus and cortex (CTX) in aged non-Tg control and 3xTg-AD mouse (a). Confocal images of double-labeling with RAGE and NeuN or Iba-1 in the hippocampus and cortex in aged non-Tg control (b) and 3xTg-AD (c) mouse. The immunoreactivity of RAGE and Iba-1-positive microglia were increased in 3xTg-AD mice compared with non-Tg controls (d, e). No differences in NeuN immunoreactivity were observed between these two groups (f). The majority of RAGE labeling overlapped with NeuN-positive neurons, although some labeling was found in nonneuronal cells (c). Data are the means \pm s.e.m. (adult: $n=6$; aged: $n=10$ ). ${ }^{*} P<0.05,{ }^{*} P<0.01$. Scale bar $=200 \mu \mathrm{m}$. 
a

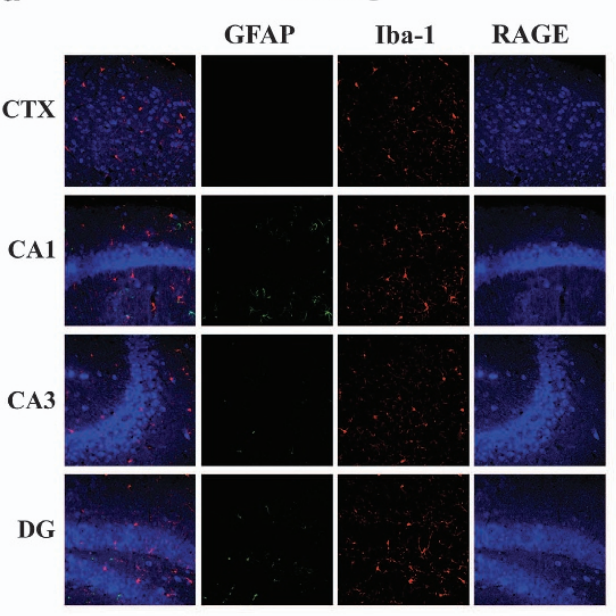

b

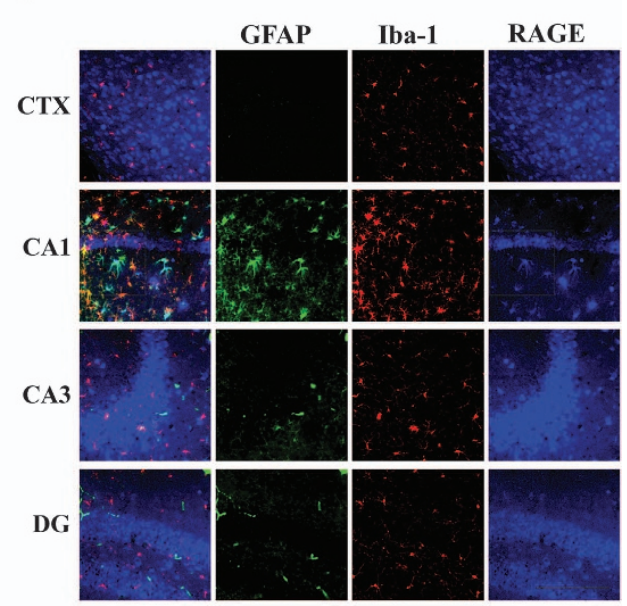

c

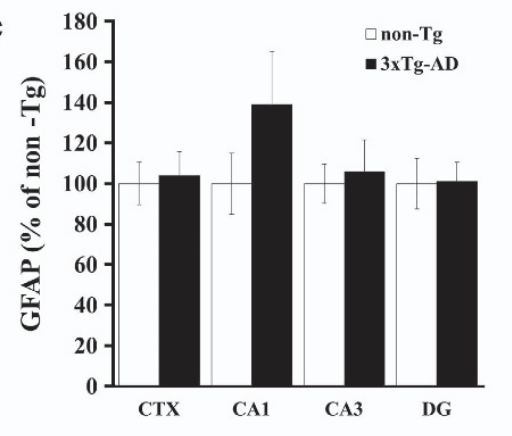

d
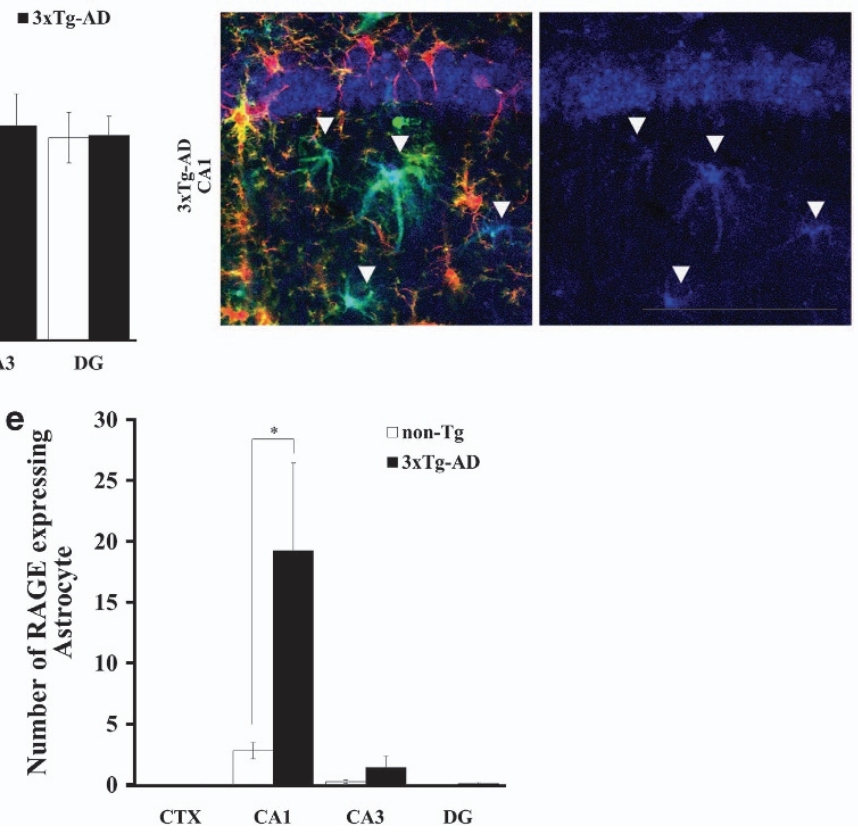

Figure 3 Co-localization of RAGE with GFAP-positive astrocytes in the hippocampal area CA1 of aged 3xTg-AD mice. Confocal images of triple-labeling with RAGE, GFAP and Iba-1 in the hippocampus and cortex (CTX) in aged non-Tg control (a) and 3xTg-AD (b) mouse. Immunoreactivity of GFAP-positive astrocytes in aged 3xTg-AD mice was not significantly different from that in aged non-Tg control mice, even though immunoreactivity in the CA1 was higher (c). However, the signals of RAGE (blue) co-localized with GFAP (green)-positive astrocytes (Iba-1 (red), d) (see reverse triangles). The number of RAGE-expressing astrocytes in the hippocampal area CA1 in aged3xTg$\mathrm{AD}$ was significantly higher than in non-Tg controls $\left(\mathbf{e},{ }^{*} P<0.05\right)$. Data are the means \pm s.e.m. (adult: $n=6 ;$ aged: $n=10$ ). Scale bar $=200 \mu \mathrm{m}$.

ANOVA of RAGE revealed that the main effects of group were significant $(\mathrm{F}(1,40)=15.21, P<0.001)$, but the main effects of region (cortex, CA1, CA3 and DG) and the significant interaction effect of group and region were not significant $(\mathrm{F}(3,40) \leqslant 0.81, P>0.49)$. Post hoc analyses revealed that significant differences between $3 \times \mathrm{Tg}-\mathrm{AD}$ and non-Tg control groups were observed in the cortex, hippocampal area CA1 and DG $(P<0.05)$ (Figure 2d).

Inflammatory responses are increased in the brains of patients with $\mathrm{AD}$ and transgenic $\mathrm{AD}$ mice, ${ }^{1}$ and correspondingly, the immunoreactivity of Iba-1, a microglial marker, was increased in 3xTg-AD mice compared with non$\mathrm{Tg}$ control mice (Figures 2b-e). Two-way ANOVA of Iba-1 immunoreactivity showed that the main effects of group were significant $(F(1,40)=27.02, P<0.001)$, but the main effects of region and the significant interaction effect of group and region were not significant $(\mathrm{F}(3,40) \leqslant 0.09, P>0.97)$. Post hoc analyses revealed that there were significant differences between $3 \mathrm{xTg}-\mathrm{AD}$ and control groups in all regions observed $(P<0.05)$ (Figure 2e). 
Notably, the immunoreactivity of NeuN, a specific marker of neurons, in 3xTg-AD mice was not different from that in non-Tg control mice (Figures 2b, c and f). Double-labeled fluorescent immunohistochemistry was conducted to localize the expression of RAGE in aged 3xTg-AD mice and aged nonTg control mice. Most RAGE-positive expression co-localized with neurons, although some expression was observed in nonneuronal cells in aged 3xTg-AD mice and non-TG control mice (Figures $2 \mathrm{~b}$ and $\mathrm{c}$ ).

To investigate the interaction of these factors, we conducted triple-labeled fluorescent staining against Iba-1, glial fibrillary acidic protein (GFAP) and RAGE to reveal the localization of RAGE expression in non-neuronal, namely glial, cells. First, expression of GFAP, which is used to measure glial reactivity, was examined in aged 3xTg-AD mice and non-Tg control mice, and its immunoreactivity was observable in the hippocampal CA1 of aged 3xTg-AD mice (Figures $3 \mathrm{a}$ and $\mathrm{b}$ ). However, two-way ANOVA revealed that the main effects of group and region (cortex, CA1, CA3, DG and stratum radiatum) were not significant $(\mathrm{F}(1,50)=2.06, \quad P=0.16$; $\mathrm{F}(4,50)=0.55, P=0.70)$, and the significant interaction effect of group and region was not significant $(\mathrm{F}(4,50)=0.55$, $P=0.70)$. Interestingly, GFAP-positive glial cells were evident in the CA1 of aged 3xTg-AD mice (Figures $3 \mathrm{a}$ and $\mathrm{b}$ ). Although there were a few instances of Iba-1-positive glial cells expressing RAGE, most of the RAGE-positive expression overlapped with GFAP-positive glial cells (Figure 3b). Specifically, RAGE-expressing astrocytes were frequently observed in the strata radiatum layer in the hippocampal area CA1 of aged $3 \mathrm{xTg}-\mathrm{AD}$ mice (Figure $3 \mathrm{~d}$ ). On the other hand, the other brain areas, such as hippocampal area CA3 and DG, showed very few RAGE-expressing astrocytes, and there were no observed instances of RAGE-expressing astrocytes in the cortex (Figure 3e). Two-way ANOVA of RAGE-positive GFAP revealed that the main effects of group were significant $(\mathrm{F}(1,40)=12.80, P<0.01)$ and that the main effects of region and the significant interaction effect of group and region were significant $(\mathrm{F}(3,40) \geqslant 10.55, \quad P<0.001)$. Post hoc analyses revealed significant differences between $3 \times \mathrm{Tg}-\mathrm{AD}$ and nonTg control mice in the hippocampal area CA1 $(P<0.05)$ (Figure 3e).

\section{Distribution and co-localization of $4 \mathrm{G} 8$ APP/A $\beta$ immunoreactivity with RAGE}

To characterize the deposition of APP and A $\beta$ in aged $3 \times$ Tg$\mathrm{AD}$ mice, tissue sections were stained with the monoclonal antibody 4G8, which detects APP and A $\beta .^{23}$ Two-way ANOVA of $4 \mathrm{G} 8$ immunoreactivity revealed that the main effects of group were significant $(\mathrm{F}(1,50)=16.16, P<0.001)$, but the main effects of region (5th layer in the cortex, non-5th layer in the cortex, CA1, CA3, and DG) and the significant interaction effect of group and region were not significant $(\mathrm{F}(4,50) \leqslant 1.06$, $P \geqslant 0.42$ ). Post hoc analyses revealed that in the cortex area of $3 x T g-A D$ mice, intracellular 4G8 labeling was significantly increased in the fifth layer and was co-localized with RAGE immunoreactivity in neuronal-shaped cells. However, in other layers of the cortex, no difference was detected in 4G8 signal compared with non-Tg control mice $(P<0.05)$ (Figure $4 \mathrm{c})$. In addition, 4G8 immunoreactivity in hippocampal areas CA1 and CA3 were significantly increased in $3 x T g-A D$ mice $(P<0.05)$ (Figure 4b). Interestingly, 4G8 labeling in the CA1 and $\mathrm{CA} 3$ of $3 \mathrm{xTg}-\mathrm{AD}$ mice was observed in both intracellular and extracellular APP/A $\beta$, but only intracellular 4G8 labeling was co-localized with RAGE $(P<0.05)$. There was no significant difference of $4 \mathrm{G} 8$ immunoreactivity in the DG of 3xTg-AD mice compared with non-Tg control mice.

\section{Distribution of HT7 human tau immunoreactivity and its co-localization with RAGE}

We examined the distribution of tau and its co-localization with RAGE in the hippocampus and cortex of aged 3xTg-AD mice using double-labeled fluorescent staining with HT7 antibody, one of the human-specific tau antibodies, and RAGE antibody (Figures 5a and b). ${ }^{17}$ Two-way ANOVA of the HT7 immunoreactivity revealed that the main effects of group were significant $(\mathrm{F}(1,40)=18.64, P<0.001)$, and the main effects of region (cortex, CA1, CA3 and DG) and the significant interaction effect of group and region were significant $(\mathrm{F}(4,40) \geqslant 10.55, \quad P \leqslant 0.001$ ) (Figure 5c). Post hoc analyses revealed that the immunoreactivity of HT7 was significantly increased only in the hippocampal area CA1 of aged 3xTg-AD mice $(P<0.05)$. In the cortex of $3 x$ Tg-AD mice, HT7 was expressed in RAGE-expressing neurons but was not significantly increased compared with non-Tg control mice. In the hippocampal area CA1 of 3xTg-AD mice, HT7 expression was co-localized with RAGE and expressed in the neuronal, but not glial, cells. Furthermore, HT7-positive neurons in hippocampal area CA1 of 3xTg-AD mice were located along the outer perimeter of the cell layer rather than inside, and HT7 labeling was observed throughout the axon. RAGE was mostly localized in neuronal cell bodies merged with signals of HT7 (Figure 5e). Signals of HT7 were almost localized in the cortical neurons of 3xTg-AD mice but were not significantly increased compared with non-Tg control mice.

Figure 4 Distribution of 4G8-positive APP/A $\beta$ and its co-localization with RAGE in aged 3xTg-AD mice. Confocal images of doublelabeling with RAGE and 4G8 in the hippocampus and cortex (CTX) in aged non-Tg control (a, d) and 3xTg-AD (b, e) mouse. Immunoreactive labeling for 4G8, a monoclonal antibody detecting APP/A 3 , was increased in aged 3xTg-AD mice compared with aged non-Tg controls (c). Notably, in the cortex, the immunoreactivity of 4G8 was significantly increased only in layer 5, whereas there were no differences in other layers (c). In the hippocampus, 4G8 immunoreactivity was significantly increased in area CA1 and CA3 but not in the DG (c). Labeling for intracellular 4G8 in neurons was co-localized with RAGE expression, whereas that for extracellular 4G8 was not (e). Data are means \pm s.e.m. (adult: $n=6$; aged: $n=10$ ). ${ }^{*} P<0.05$. Scale bar $=100 \mu \mathrm{m}$. 
a

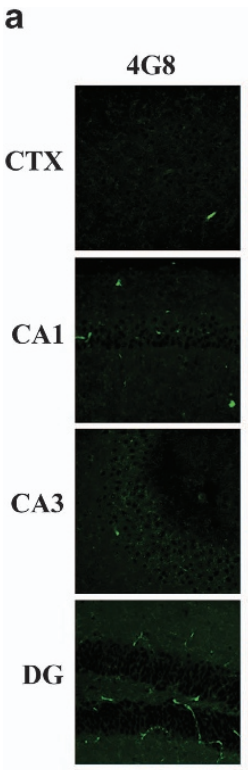
Non-Tg RAGE
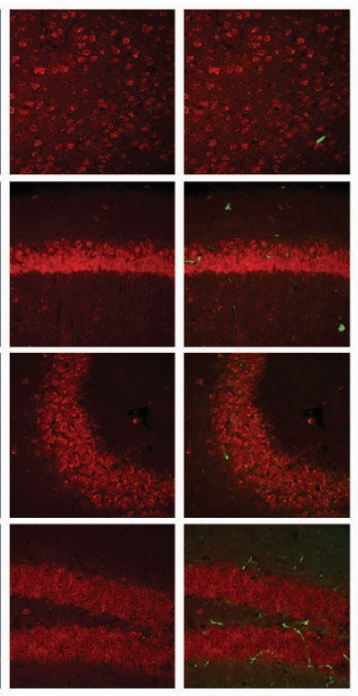

b
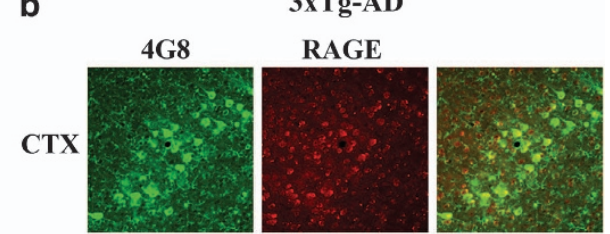

CA1
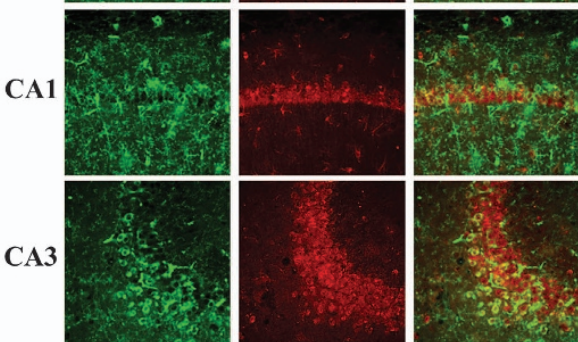

C $\mathbf{3 0 0}$
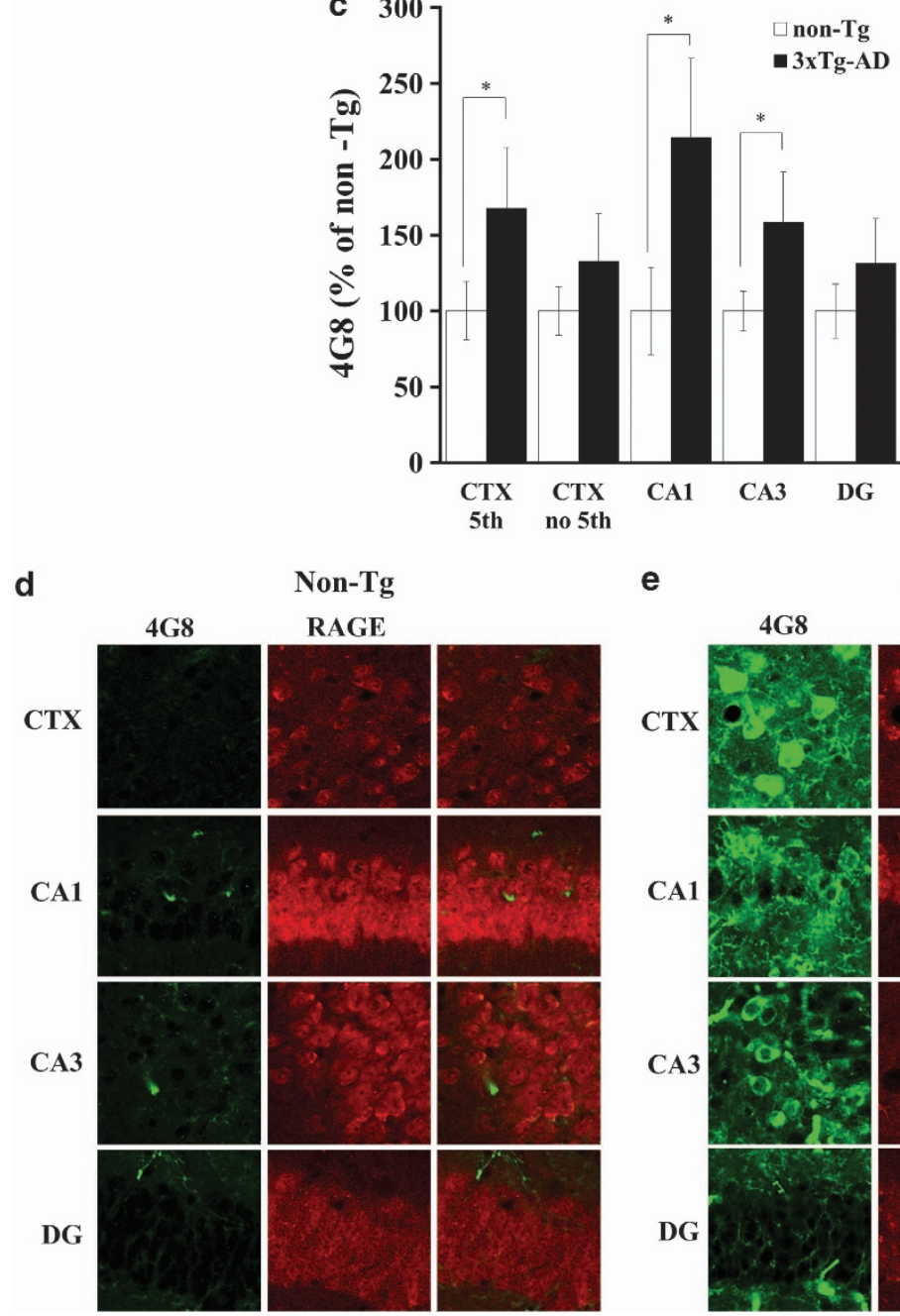

\section{e}

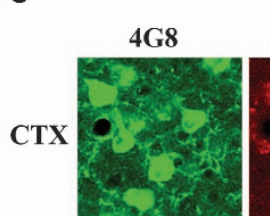

3xTg-AD

RAGE
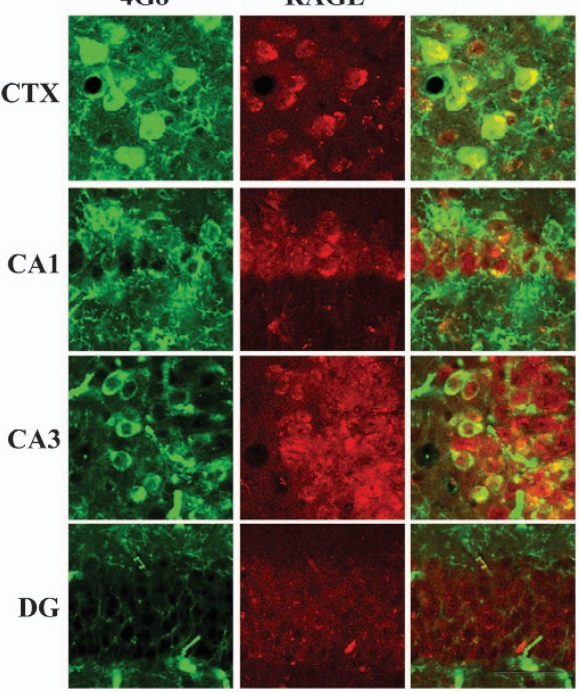


\section{DISCUSSION}

Recent research efforts have been focused on understanding the molecular and cellular mechanisms underlying age-related neurodegenerative disorders. $\mathrm{AD}$ is the most common neurodegenerative disorder and is associated with SPs and NFTs in the brain. Advances in transgenic technology have established animal models exhibiting one or both of the main pathological hallmarks of $\mathrm{AD}$, such as SPs and NFTs. However, clinical symptoms in humans have not always been paralleled in animal models. Using the 3xTg-AD mouse model, which exhibits the full spectrum of $\mathrm{AD}$ neuropathology, ${ }^{5,17}$ the present study was conducted to examine age-dependent changes in and the cellular localizations of RAGE as well as the co-localization between RAGE and SPs or NFTs.

Immunohistochemical studies with human tissues have demonstrated that RAGE levels are increased in the brains of patients with $\mathrm{AD}$ in comparison with those of aged-matched controls, ${ }^{11-13,21}$ and a similar increase in RAGE expression has been reported in transgenic $\mathrm{AD}$ mice with overexpression of the Swedish mutant form of APP. ${ }^{15}$ In parallel, the role of RAGE in the pathological processes of $\mathrm{AD}$ has been the focus of many studies attempting to reveal the mechanisms underlying cellular dysfunction. ${ }^{9,14,24-26}$ In accord with the results of these investigations, the present study demonstrated that RAGE expression was increased in the cortex and hippocampus of the aged 3XTg-AD mice (22-24 months old).

Several transgenic AD mouse models have been developed for studying the cellular mechanisms of $\mathrm{AD}$ pathogenesis and/ or drug development. Most of these models express mutant forms of genes linked to early-onset familial AD, such as APP, PS or tau, ${ }^{27}$ and provide invaluable contributions to the studies related to $\mathrm{AD}$ but do not mimic the full spectrum of $\mathrm{AD}$ pathology, including the SPs and NFTs. To overcome this, a $3 \mathrm{xTg}-\mathrm{AD}$ mouse model was generated to express the three major genes associated with familial $\mathrm{AD}$, namely APP, presenilin1 and tau. 3xTG-AD mice show AD-like SPs and NFTs. ${ }^{5,17}$ In addition to these markers of $\mathrm{AD}$, RAGE is reported to have a role in amyloidosis and tau pathology. ${ }^{9,14,28}$ Therefore, to determine a role of RAGE in the 3xTg-AD mouse model, representing the full spectrum of $\mathrm{AD}$ neuropathology, the present study examined the distribution and cellular localization of RAGE in aged 3xTg-AD and non-Tg control mice.

Using double- or triple-labeled fluorescent immunohistochemistry, we observed that RAGE expression was increased in the aged $3 \times \mathrm{Tg}-\mathrm{AD}$ mice and that the cellular localization was mostly in neurons, although in rare instances, RAGE expression was observed in non-neuronal cells. In aged 3xTg-AD mice, expression of Iba-1, a microglial marker, was also increased and showed very little overlap with RAGE expression. Consequently, we investigated whether the RAGE-positive non-neuronal cells were astrocytes or microglial cells. Triple-labeled fluorescent immunohistochemistry revealed that some RAGE-positive non-neuronal cells were located in GFAPpositive astrocytes, and very few RAGE-positive non-neuronal cells were located in Iba-1-positive microglial cells. However, because levels of Iba-1 expression were increased in aged 3xTg$\mathrm{AD}$ mice in the present study and because RAGE-dependent signaling in microglia reportedly play a role in the pathogenesis of an inflammatory response and $\mathrm{A} \beta$ accumulation, ${ }^{25,29,30}$ it will be interesting to measure the expression levels of microglial RAGE in aged $3 \times \mathrm{xTg}-\mathrm{AD}$ mice with several markers more specific to microglia and to elucidate the role of microglial RAGE in amplifying the neuronal toxicity of beta amyloid in the presence of activated microglia.

AGEs, ligands of RAGE, accumulate in $\mathrm{AD}$ brains and contribute to amyloidosis. ${ }^{28}$ Moreover, studies demonstrating a role of RAGE in $A \beta$ transport have shown that RAGE is involved in $A \beta$ transport across the blood-brain barrier ${ }^{24}$ and in the intraneuronal transport of $\mathrm{A} \beta .^{26}$ Arancio et al., ${ }^{14}$ who studied the effects of RAGE on $A \beta$-induced impairment of neuronal function, reported that neuronal overexpression of RAGE in mice with mutant APP exacerbated impairments of spatial memory and synaptic plasticity. In 3xTg-AD mice, $4 \mathrm{G} 8$-positive immunoreactivity of APP/A $\beta$ was observed in neurons and in the extracellular regions, which is consistent with a previous report. ${ }^{3}$ Further, 4G8-positive APP/A $\beta$ neurons co-localized with RAGE-positive immunoreactivity. However, to conclude that the RAGE signal is not co-localized with extracellular $A \beta$ plaque in aged $3 x T g-A D$ mice, association of RAGE with extracellular $\mathrm{A} \beta$ plaques must be examined in the hippocampus and cortex of $3 \times \mathrm{Tg}-\mathrm{AD}$ mice using other markers to detect extracellular $A \beta$ plaques. However, one study has reported that extracellular $A \beta$ plaques examined by thioflavin-S staining were observed only in the entorhinal cortex. ${ }^{5}$

The finding that AGEs are co-localized with paired helical filament tau suggests an intrinsic link of AGEs with tau, and a recent study revealed that AGE-induced tau hyperphosphorylation impairs synaptic plasticity and spatial memory through RAGE. ${ }^{9,10}$ In the $3 x \mathrm{Tg}-\mathrm{AD}$ mice, expression of HT7-positive human tau was increased in the cortex and hippocampus, particularly in the CA1 region, where HT7-positive human tau immunoreactivity co-localized with labeling for RAGE.

The pathological features of $\mathrm{AD}$ are $\mathrm{A} \beta$ accumulation, tau protein hyperphosphorylation and increased inflammatory activity in the cortex and hippocampus., ${ }^{9}$ RAGE

Figure 5 Distribution of HT7-positive human tau protein and its co-localization with RAGE in aged 3xTg-AD mice. Confocal images of double-labeling with RAGE and HT7 in the hippocampus and cortex (CTX) in aged non-Tg control (a, d) and 3xTg-AD (b, e) mice. Immunoreactivity of HT7, a marker of human tau protein, was higher in the hippocampal area CA1 of aged 3xTg-AD mice compared with aged non-Tg control (c). HT7 labeling overlapped with RAGE. In aged 3xTg-AD mice, HT7 was expressed in some cortical neurons, CA1 neurons, and some axons of the mossy fiber, whereas the DG and hippocampal area CA3 did not show immunoreactivity for HT7 when

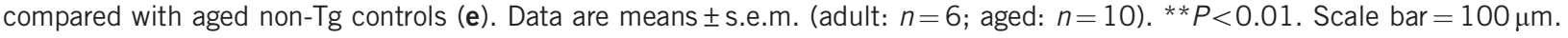


a

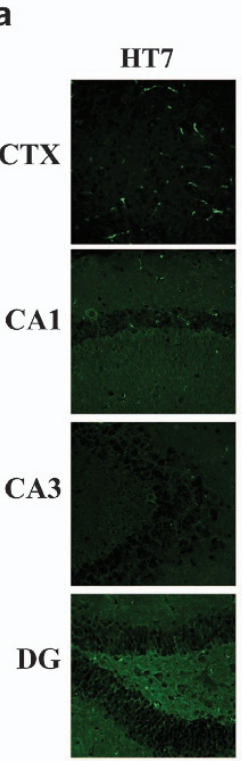

b

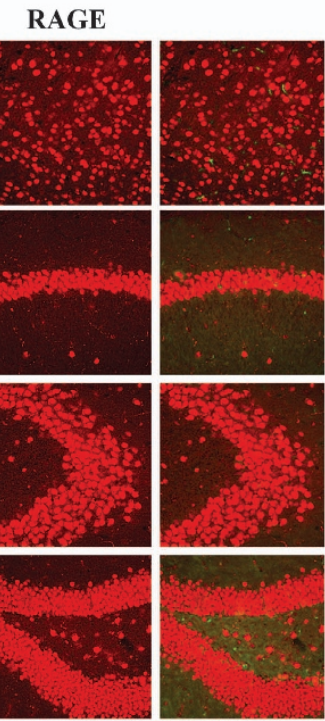

HT7

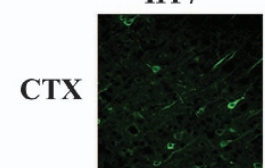

3xTg-AD

CA1
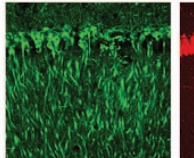

CA3
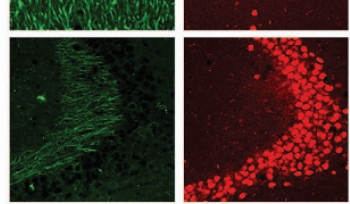

DG
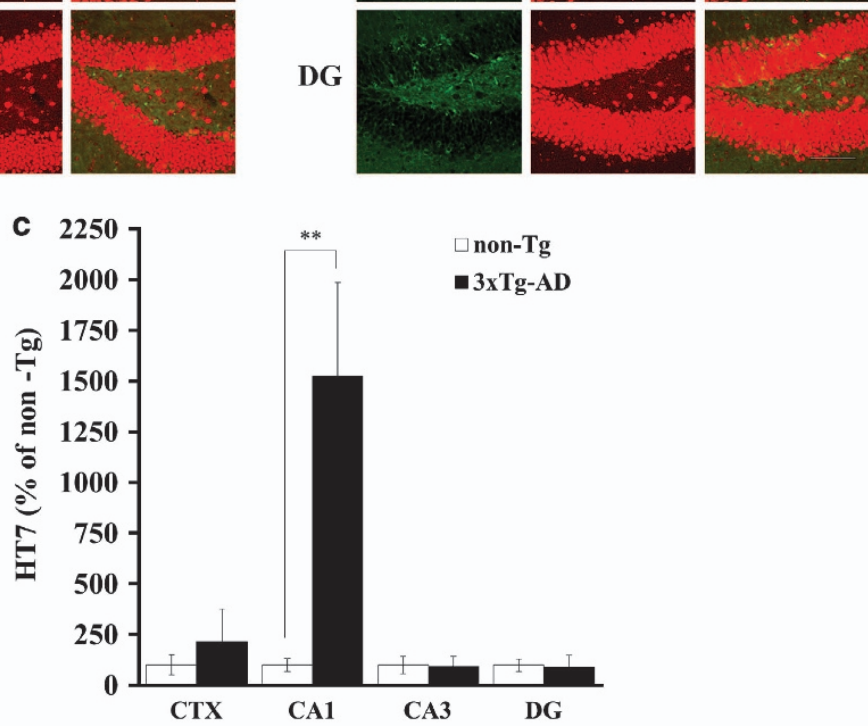

d Non-Tg


CA3



DG
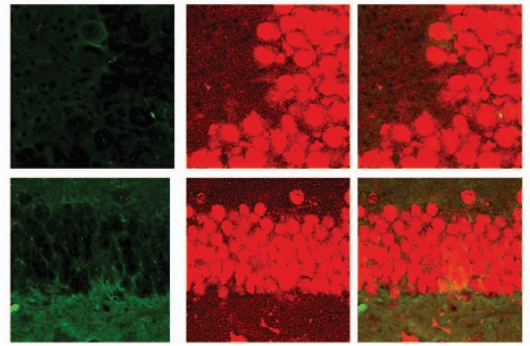

e

3xTg-AD
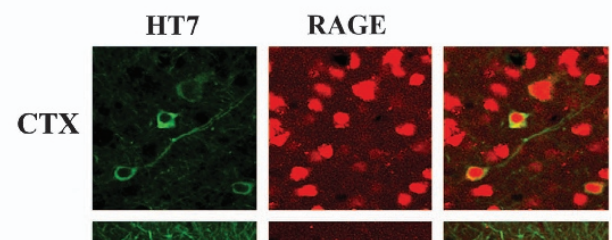

CA1
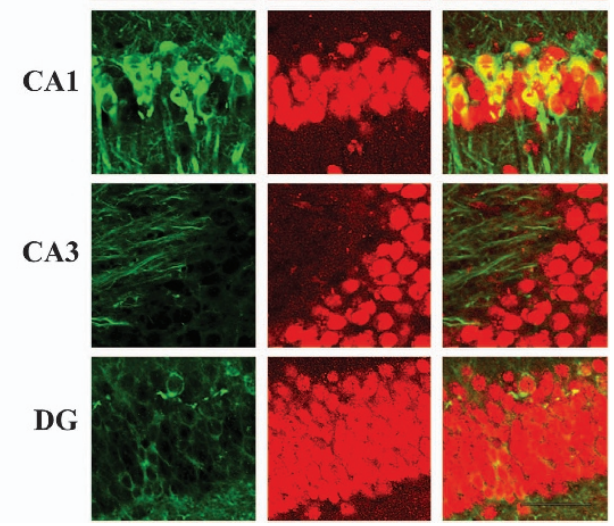
and components of its signaling pathway have been shown to interact with $\mathrm{A} \beta$ and have a key role in tau hyperphosphorylation. Furthermore, increased RAGE expression is found at sites of inflammation in $\mathrm{AD}$ brains. ${ }^{18}$ Thus, because RAGE expression seems to be affected by several characteristics observed in $\mathrm{AD}$ brains, the 3xTg-AD mouse model, which encompasses all these characteristics, is a better model for studying RAGE mechanisms and developing therapeutic drugs. The results of the present study, such as the distribution of RAGE, cellular localization and its co-localization with $A \beta$ or tau, in 3xTg-AD mice might be very informative in studying the interaction of RAGE with key players in AD pathology, and this model may prove useful for evaluating drug candidates, such as RAGE inhibitors, and interventions for $A \beta$ accumulation and tau hyperphosphorylation.

\section{CONFLICT OF INTEREST}

The authors declare no conflict of interest.

\section{ACKNOWLEDGEMENTS}

This work was supported by a grant (Kiom-2010-2) from the InterInstitutional Collaboration Research Program provided by the Korea Research Council of Fundamental Science \& Technology (KRCF) and a Korea Research Foundation Grant funded by the Korean Government (KRF-2010-0023880).

1 Mattson MP. Pathways towards and away from Alzheimer's disease. Nature 2004; 430: 631-639.

2 Selkoe DJ. Alzheimer's disease: genes, proteins, and therapy. Physioll Rev 2001; 81: 741-766.

3 Billings LM, Oddo S, Green KN, McGaugh JL, LaFerla FM. Intraneuronal Abeta causes the onset of early Alzheimer's disease-related cognitive deficits in transgenic mice. Neuron 2005; 45: 675-688.

4 LaFerla FM, Green KN, Oddo S. Intracellular amyloid-beta in Alzheimer's disease. Nat Rev Neurosci 2007; 8: 499-509.

5 Oddo S, Caccamo A, Kitazawa M, Tseng BP, LaFerla FM. Amyloid deposition precedes tangle formation in a triple transgenic model of Alzheimer's disease. Neurobiol Aging 2003; 24: 1063-1070.

6 Grundke-Iqbal I, Iqbal K, Quinlan M, Tung YC, Zaidi MS, Wisniewski HM Microtubule-associated protein tau. A component of Alzheimer paired helical filaments. J Biol Chem 1986; 261: 6084-6089.

7 Schmidt AM, Hori O, Cao R, Yan SD, Brett J, Wautier JL et al. RAGE: a novel cellular receptor for advanced glycation end products. Diabetes 1996; 45(Suppl 3), S77-S80.

8 Yan SD, Chen X, Fu J, Chen M, Zhu H, Roher A et al. RAGE and amyloid-beta peptide neurotoxicity in Alzheimer's disease. Nature 1996; 382: 685-691.

9 Li XH, Lv BL, Xie JZ, Liu J, Zhou XW, Wang JZ. AGEs induce Alzheimer-like tau pathology and memory deficit via RAGE-mediated GSK-3 activation. Neurobiol Aging 2012; 33: 1400-1410.

10 Yan SD, Chen X, Schmidt AM, Brett J, Godman G, Zou YS et al. Glycated tau protein in Alzheimer disease: a mechanism for induction of oxidant stress. Proc Natl Acad Sci USA 1994; 91: 7787-7791.

11 Lue LF, Walker DG, Brachova L, Beach TG, Rogers J, Schmidt AM et al. Involvement of microglial receptor for advanced glycation endproducts (RAGE) in Alzheimer's disease: identification of a cellular activation mechanism. Exp Neurol 2001; 171: 29-45.

12 Sasaki N, Toki S, Chowei H, Saito T, Nakano N, Hayashi $\mathrm{Y}$ et al. Immunohistochemical distribution of the receptor for advanced glycation end products in neurons and astrocytes in Alzheimer's disease. Brain Res 2001; 888: 256-262.

13 Miller MC, Tavares R, Johanson CE, Hovanesian V, Donahue JE, Gonzalez L et al. Hippocampal RAGE immunoreactivity in early and advanced Alzheimer's disease. Brain Res 2008; 1230: 273-280.

14 Arancio O, Zhang HP, Chen X, Lin C, Trinchese F, Puzzo D et al. RAGE potentiates Abeta-induced perturbation of neuronal function in transgenic mice. EMBO J 2004; 23: 4096-4105.

15 Cho HJ, Son SM, Jin SM, Hong HS, Shin DH, Kim SJ et al. RAGE regulates BACE1 and Abeta generation via NFAT1 activation in Alzheimer's disease animal model. FASEB J 2009; 23: 2639-2649.

16 LaFerla FM, Oddo S. Alzheimer's disease: Abeta, tau and synaptic dysfunction. Trends Mol Med 2005; 11: 170-176.

17 Oddo S, Caccamo A, Shepherd JD, Murphy MP, Golde TE, Kayed R et al. Triple-transgenic model of Alzheimer's disease with plaques and tangles: intracellular Abeta and synaptic dysfunction. Neuron 2003; 39: 409-421.

18 Leclerc E, Sturchler E, Vetter SW, Heizmann CW. Crosstalk between calcium, amyloid beta and the receptor for advanced glycation endproducts in Alzheimer's disease. Rev Neurosci 2009; 20: 95-110.

19 Bialowas-McGoey LA, Lesicka A, Whitaker-Azmitia PM. Vitamin E increases S100B-mediated microglial activation in an S100B-overexpressing mouse model of pathological aging. Glia 2008; 56: 1780-1790.

20 Silverberg GD, Miller MC, Messier AA, Majmudar S, Machan JT, Donahue JE et al. Amyloid deposition and influx transporter expression at the bloodbrain barrier increase in normal aging. J Neuropathol Exp Neurol 2010; 69: 98-108.

21 Donahue JE, Flaherty SL, Johanson CE, Duncan JA 3rd, Silverberg GD, Miller MC et al. RAGE, LRP-1, and amyloid-beta protein in Alzheimer's disease. Acta Neuropathol 2006; 112: 405-415.

$22 \mathrm{Han}$ SH, Kim YH, Mook-Jung I. RAGE: the beneficial and deleterious effects by diverse mechanisms of actions. Mol Cells 2011; 31: 91-97.

23 Borchelt DR, Ratovitski T, van Lare J, Lee MK, Gonzales V, Jenkins NA et al. Accelerated amyloid deposition in the brains of transgenic mice coexpressing mutant presenilin 1 and amyloid precursor proteins. Neuron 1997; 19: 939-945.

24 Deane R, Du Yan S, Submamaryan RK, LaRue B, Jovanovic S, Hogg E et al. RAGE mediates amyloid-beta peptide transport across the blood brain barrier and accumulation in brain. Nat Med 2003; 9: 907-913.

25 Origlia N, Righi M, Capsoni S, Cattaneo A, Fang F, Stern DM et al. Receptor for advanced glycation end product-dependent activation of p38 mitogen-activated protein kinase contributes to amyloid-beta-mediated cortical synaptic dysfunction. J Neurosci 2008; 28: 3521-3530.

26 Takuma K, Fang F, Zhang W, Yan S, Fukuzaki E, Du H et al. RAGEmediated signaling contributes to intraneuronal transport of amyloid-beta and neuronal dysfunction. Proc Natl Acad Sci USA 2009; 106: 20021-20026.

27 Duff K, Suleman F. Transgenic mouse models of Alzheimer's disease: how useful have they been for therapeutic development? Brief Funct Genomic Proteomic 2004; 3: 47-59.

28 Vitek MP, Bhattacharya K, Glendening JM, Stopa E, Vlassara H, Bucala R et al. Advanced glycation end products contribute to amyloidosis in Alzheimer disease. Proc Natl Acad Sci USA 1994; 91: 4766-4770.

29 Fang F, Lue LF, Yan S, Xu H, Luddy JS, Chen D et al. RAGE-dependent signaling in microglia contributes to neuroinflammation, Abeta accumulation, and impaired learning/memory in a mouse model of Alzheimer's disease. FASEB J 2010; 24: 1043-1055.

30 Origlia N, Bonadonna C, Rosellini A, Leznik E, Arancio O, Yan SS et al Microglial receptor for advanced glycation end product-dependent signal pathway drives beta-amyloid-induced synaptic depression and long-term depression impairment in entorhinal cortex. J Neurosci 2010; 30: 11414-11425.

(1) (2) This work is licensed under a Creative Commons Attribution-NonCommercial-ShareAlike 3.0 Unported License. To view a copy of this license, visit http:// creativecommons.org/licenses/by-nc-sa/3.0/ 